\title{
Fractional calculus model of articular cartilage based on experimental stress-relaxation
}

\author{
P.A. Smyth ${ }^{1}$ - I. Green ${ }^{1}$
}

Received: 8 January 2014 / Accepted: 24 February 2015 / Published online: 10 March 2015

(C) Springer Science+Business Media Dordrecht 2015

\begin{abstract}
Articular cartilage is a unique substance that protects joints from damage and wear. Many decades of research have led to detailed biphasic and triphasic models for the intricate structure and behavior of cartilage. However, the models contain many assumptions on boundary conditions, permeability, viscosity, model size, loading, etc., that complicate the description of cartilage. For impact studies or biomimetic applications, cartilage can be studied phenomenologically to reduce modeling complexity. This work reports experimental results on the stress-relaxation of equine articular cartilage in unconfined loading. The response is described by a fractional calculus viscoelastic model, which gives storage and loss moduli as functions of frequency, rendering multiple advantages: (1) the fractional calculus model is robust, meaning that fewer constants are needed to accurately capture a wide spectrum of viscoelastic behavior compared to other viscoelastic models (e.g., Prony series), (2) in the special case where the fractional derivative is $1 / 2$, it is shown that there is a straightforward time-domain representation, (3) the eigenvalue problem is simplified in subsequent dynamic studies, and (4) cartilage stress-relaxation can be described with as few as three constants, giving an advantage for large-scale dynamic studies that account for joint motion or impact. Moreover, the resulting storage and loss moduli can quantify healthy, damaged, or cultured cartilage, as well as artificial joints. The proposed characterization is suited for high-level analysis of multiphase materials, where the separate contribution of each phase is not desired. Potential uses of this analysis include biomimetic dampers and bearings, or artificial joints where the effective stiffness and damping are fundamental parameters.
\end{abstract}

Keywords Articular cartilage · Fractional calculus · Unconfined compression · Relaxation · Storage and loss modulus · Viscoelasticity

$\triangle$ P.A. Smyth

pasmyth4@gatech.edu

I. Green

itzhak.green@me.gatech.edu

1 School of Mechanical Engineering, Georgia Institute of Technology, Atlanta, GA 30332, USA 


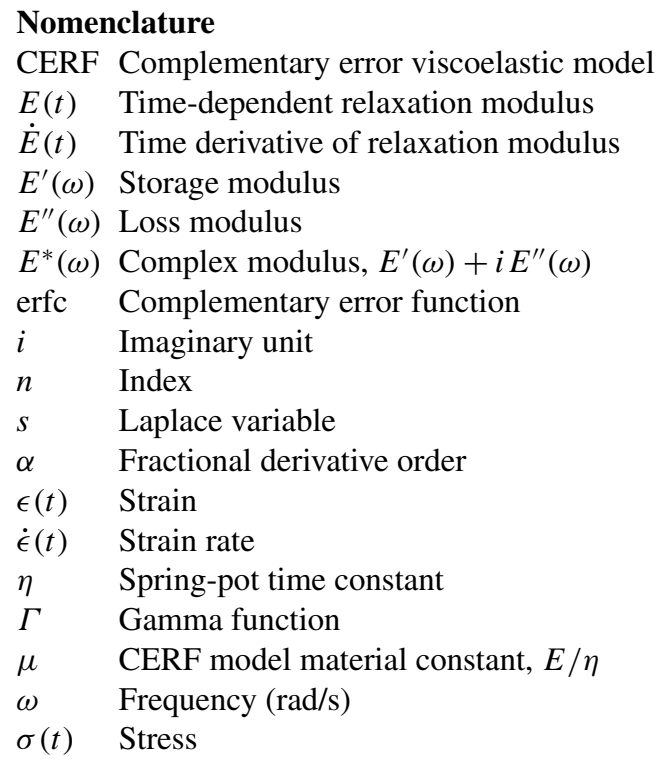

\section{Introduction}

Articular cartilage facilitates motion in joints while providing compressive load support. The porous, biphasic (solid-fluid) structure of cartilage has been studied for many decades. This has led to advances in constitutive modeling, artificial joint replacements, and the characterization of osteoarthritis. Mechanical tests are performed to corroborate the prevailing biphasic and triphasic (solid-fluid-ionic) models. A variety of experiments are used to test cartilage. In stress-relaxation experiments, cartilage displays elastic and dissipative mechanisms. These mechanisms are also central in viscoelastic materials, which are prevalent in traditional mechanical systems. Interesting applications for biomimetic materials, based on cartilage, arise from the study of biphasic materials. These include flexible mechanical bearings in rotordynamic systems (Grybos 1991; Friswell 2007) and improved porous bearings in industrial applications (Elsharkawy and Nassar 1996).

A great deal of cartilage research has focused on the interactions of the collagen matrix and the lubricating synovial fluid that permeates the joint capsule (Charnley 1960; McCutchen 1962; Ateshian 2009; Ateshian et al. 1997, 1998). The prevailing constitutive theories account for the biphasic and triphasic properties of cartilage (Mow et al. 1980; Lai et al. 1981; Armstrong et al. 1984; Lai et al. 1991). These models are physiologically comprehensive; however, they do not typically match experimental results well. With an eye toward biomimetics, the solid and fluid interactions of cartilage can be viewed holistically. Therefore, the total response includes the solid and fluid phases and their interactions. These interactions include frictional drag between the solid and fluid phases, compressibility of the solid matrix, or other mechanisms. Mechanical systems with mechanisms for energy storage and dissipation work well in this application. The models are phenomenological and based on stress-relaxation experiments. Typically, phenomenological models use fewer parameters, and they do not associate directly to a specific structure or location within the cartilage body. Therefore, the cartilage behavior can be compared in a broad sense. This allows for a straightforward and convenient comparison between samples for any number of metrics 
such as health, age, weight, use, breed, etc. Comparisons between joints can easily be made, as well as between healthy and osteoarthritic cartilage. In addition, the phenomenological model is applicable over a wide spectrum of relaxation behavior (Lakes 1998). This is particularly advantageous when compared to the small bandwidth captured by many biphasic models.

Coletti et al. (1972) and Parsons and Black (1977) have previously used spring and damper models to characterize cartilage. However, these linear models did not match the experimental results from creep tests. In particular, Coletti et al. determined that cartilage exhibits nonlinear viscoelastic behavior dependent on strain. The work was performed around the time that the biphasic models were developing. The biphasic models (Mow et al. 1980; Lai et al. 1981; Armstrong et al. 1984; Lai et al. 1991; Mak 1986) began to dominate the research landscape, even as Woo et al. (1980) (using Fung's model (1967) for soft tissue) reached favorable comparisons with the relaxation experiments of Mow (1977). Simon et al. (1984) compared the spring and damper and biphasic models under stress-relaxation. The work highlights the differences of the model theories. The spring and damper models are advantageous on the macroscale; however, they cannot separate the contributions of the solid and fluid phases.

More recent research from Wang (1997), Ehlers and Markert (2000, 2001), and Wilson et al. $(2004,2005)$ uses various spring and damper representations to model the fibril part of cartilage. The poroviscoelastic fibril reinforced model developed by Wilson et al. considers the local morphology of collagen fibers and their apparent strong influence on stress and strain (the springs are strain-dependent, or nonlinear). Wilson's work compares favorably to DiSilvestro and Suh's (2001). Garcia et al. (2006) uses a similar model to Wilson's $(2004,2005)$ to describe the solid portion of the nonlinear biphasic model. Finally, Julkunen et al. (2008) corroborates the work of Wilson et al. (2004, 2005) with a FEM study, finding good agreement between the experiment and model in stress-relaxation applications. These theories are the leading constitutive models for cartilage, and additional information is available from Mow et al. (1993, 2005). The proposed fractional calculus model is not a replacement for the aforementioned models, but rather a high-level characterization of biphasic behavior that offers inherent advantages and utility in large system analysis. The fractional model is proven as a capable viscoelastic model (Carpinteri and Mainardi 1997; Mainardi and Spada 2011) and has many advantages in biomechanical applications and biomimetics (Magin 2006). These typically include model succinctness and a certain compatibility with conventional calculus and integer-order differential equations that many engineers and scientists are familiar with (West et al. 2003).

Stress-relaxation tests and mechanical models have been used in cartilage research for many decades. The spring and dashpot models are simplifications of the actual biphasic behavior of cartilage; however, there is a need for such models (Argatov 2013). The utility of a spring/dashpot model is apparent in larger-scale studies, e.g., when cartilage is incorporated into an impact study. Here, phenomenological models may be better suited for analysis. Argatov (2013) notes that the spring/dashpot, or viscoelastic, models are widely applicable as they capture the behavioral characteristics of cartilage. A trade-off exists between model complexity and the comprehensive description of phases and their interactions. In certain applications (e.g., joint dynamics), the phenomenological models are better suited for study. The recent paper by Tanaka et al. (2014) is one such example of the utility of the phenomenological models. The authors use a standard linear solid to characterize relaxation behavior of cartilage in multiple joints. The results show that the mechanical properties of cartilage may be region-specific within the joint. The model proposed herein is applicable to this type of study. 
Whereas the viscoelastic models are not masquerading as detailed models for cartilage, their utility lies in the inclusive description that they provide. Fractional calculus is already well established as a robust model for viscoelastic behavior (Bagley and Torvik 1979, 1983, 1985, 1986; Rogers 1983; Koeller 1984; Torvik and Bagley 1984; Koeller 1986; Bagley 1989). Moreover, it will be shown herein that a special case of the fractional calculus model is capable of describing the stress-relaxation behavior of cartilage not only in the frequency domain, but also in the time-domain. This special case occurs when the fractional derivative is one-half, $\alpha=1 / 2$, and results in the complementary error function (CERF) model (Szumski and Green 1991; Szumski 1993). There are multiple benefits of the CERF model: (1) a succinct time-domain solution is readily available for fitting experimental data (this is not the case for any other fractional calculus derivative order), (2) with an expansion, the CERF model requires only a polynomial fit in the time-domain, (3) the time and frequency domains are analytically linked by the elastic-viscoelastic correspondence principle (whereas this is true of the fractional calculus model for any derivative order, the time-domain representation of the CERF makes this particularly useful), (4) the frequency-dependent storage and loss moduli are obtained directly from time-domain stress-relaxation experiments, (5) the CERF model uses few (as little as three) constants to robustly and accurately describe cartilage over large time-spans or frequency decades, and (6) the CERF model is compact for use in large-scale studies, where a biphasic material (e.g., cartilage) is only one component of a much larger system. These advantages are important for biomimetic and impact studies, where the comprehensive models may be too intricate in the analysis. The current study indicates that the CERF model is quite capable of describing cartilage relaxation. This model is developed herein, following a discussion of the experimental procedures.

The mechanical properties of cartilage are determined in many ways. The techniques developed by Mow et al. (1980), Eisenfeld et al. (1978), and Mow and Mansour (1977) using confined compression are prevalent today. These techniques include a rigid cylindrical chamber that prevents fluid from flowing in the horizontal direction. Confined compression requires special loading routines to allow fluid to travel in the vertical direction. Figure 1(a) shows how a pseudo-relaxation experiment is performed in a confined compression. The hydrated sample is forced in the vertical direction into a porous indenter. The tests require a ramp displacement loading (approximately $2 \mathrm{~s}$ ) to allow fluid to permeate the indenter or punch (Fig. 1(b)). However, a ramp is not a common phenomenological load for cartilage, and a "true" stress-relaxation experiment cannot be performed in confined compression. This is because the confining chamber creates super high stresses (hydrostatic pressure) in the cartilage plugs when an instantaneous displacement is attempted. The confining chamber restrains cartilage along the walls, which introduces a 3D stress field on the cartilage plug.

In the unconfined case, a super fast (practically instantaneous) displacement can be physically imposed on the cartilage sample, as shown in Fig. 1(d). This is a classical relaxation case to a step strain. Precisely such a test is needed to directly extract the storage and loss moduli via the Boltzmann superposition principle (discussed herein). Hence, unconfined compression offers multiple advantages over the confined compression used in prior studies (Mow et al. 1980; Eisenfeld et al. 1978; Mow and Mansour 1977). With an eye toward biomimetics, unconfined compression offers clarity to the characterization of multiphase composites. It can be postulated that unconfined compression is likely to dictate a lower bound for biphasic material properties, whereas confined compression produces an upper bound. 


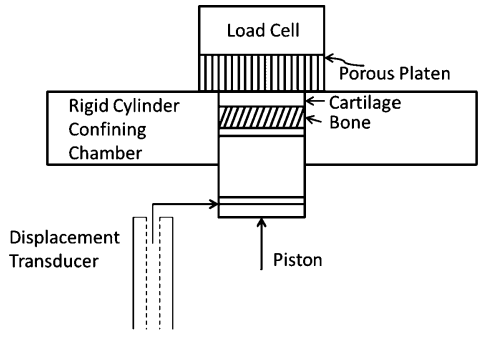

(a) Reproduction of test setup from Mow et al (1980); Eisenfeld et al (1978); Mow and Mansour (1977)

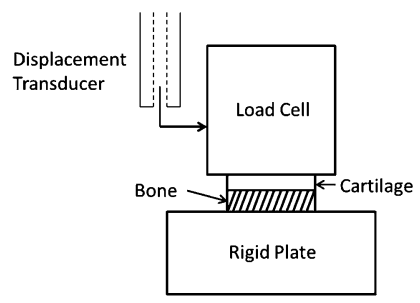

(c) Schematic of test setup in current study

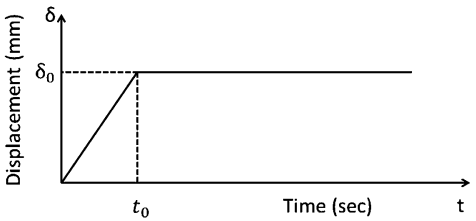

(b) Reproduction of ramp input from Mow et al (1980); Eisenfeld et al (1978); Mow and Mansour (1977)

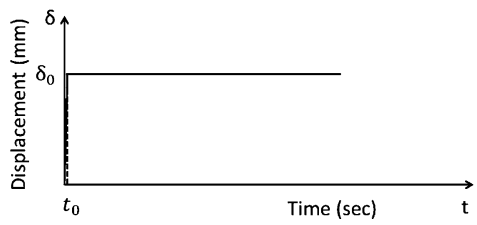

(d) Instantaneous displacement used in current study

Fig. 1 Comparison of experimental setups for measuring cartilage

\section{Materials and methods}

In this study, articular cartilage samples are harvested from the right stifle joints of horses that are euthanized for other reasons. Equine samples are used for multiple reasons: the cartilage surfaces are large and allow for "macroscale" analysis, the joints carry large loads (meaning that there are typically higher stresses within the joints), and the availability of samples is suitable. In addition, equine and human articular cartilage have similar structural features and collagen organization (Malda et al. 2012).

After euthanasia, intact joints are removed from the horses. The joints remain sealed in their native joint capsule until they are needed for analysis. The cartilage is harvested by dissection of the surrounding tissue and resized with an industrial bandsaw. The cartilage surface is hydrated with a saline solution $(0.9 \%)$ to prevent drying.

The medial condyle of the right rear stifle is used for study. The stifle joint is mechanically analogous to the human knee, and the condyle contains an area of thick and relatively flat cartilage (approximately $1.5-3 \mathrm{~mm}$ thick). After bulk harvesting and resizing of the condyle, a 10-mm-diameter plug is created with a hollow punch. The punch is driven into the sample with an arbor press, depicted in Fig. 2. With the punch embedded in the cartilage and subchrondral bone, the surrounding cartilage is removed with a rotary device. The punch has an access hole to allow for hydration of the sample. After the plug is created, it is immersed in saline. The average time from the beginning of dissection to immersion is less than $20 \mathrm{~min}$. The joint capsule is open for approximately $10 \mathrm{~min}$ during the process. Previous research highlights the importance of minimizing the exposure time of cartilage to open-air (Smyth et al. 2014). 
Fig. 2 Schematic of the $10 \mathrm{~mm}$ plug creation process

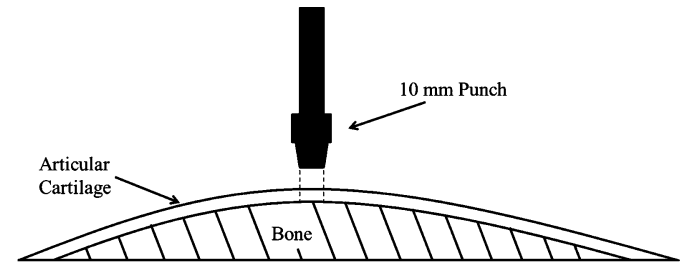

(a) Side schematic of stifle joint before plug is created

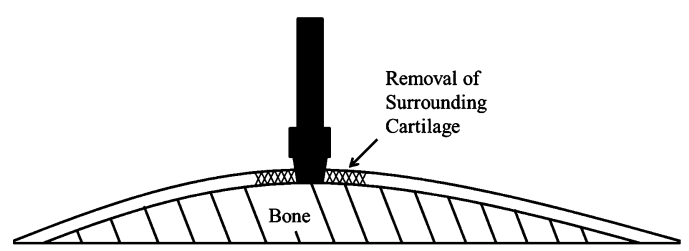

(b) Punch embedded in the cartilage as the surrounding structure is removed

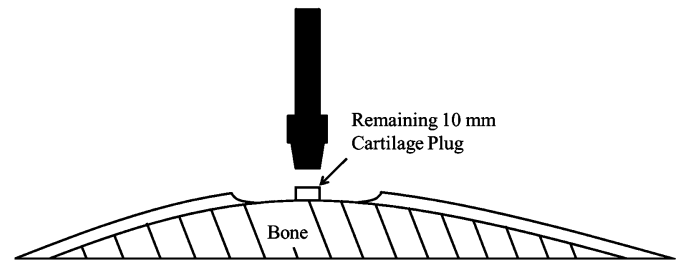

(c) The remaining cartilage plug

The cartilage plugs are placed in a UMT CETR tribometer. The tribometer imposes a nearly instantaneous (within approximately $30 \mathrm{~ms}$ ) displacement on the cartilage surface, while tracking the force generated in the cartilage matrix. By design, this is a stressrelaxation experiment. The tribometer holds a $12-\mathrm{mm}$ rigid aluminum cylinder attached to a load cell, as shown in Fig. 3. Initially, the cylinder contacts the cartilage surface with a preload of $0.5 \mathrm{~N}$. The preload ensures that the cylinder makes complete contact with the cartilage surface. In effect, the preload is flattening out any curvature in the cartilage. At time $t=0$, a practically instantaneous downward displacement is imposed on the cartilage, and the resulting force is measured. After approximately $180 \mathrm{~s}$ of measurement, the rigid cylinder is withdrawn from the surface. The cartilage is allowed two minutes to recover between tests, and the procedure is repeated. Testing indicated that the recovery time was sufficient (where additional time did not change the results). The typical test includes four runs at a lower strain, followed by four runs at a higher strain. The tribometer samples at $1000 \mathrm{~Hz}$, which yields approximately 180,000 data points for each run.

Typically, displacements of $0.25 \mathrm{~mm}$ and $0.35 \mathrm{~mm}$ are imposed on the cartilage matrix. The cartilage thickness is measured after the relaxation experiment because the thickness measurement is destructive to the sample. Therefore, a priori strains cannot be determined, but the displacements of $0.25 \mathrm{~mm}$ and $0.35 \mathrm{~mm}$ are designed to strain the cartilage matrix from 5-15\%. The bulk of the relaxation behavior has occurred within $180 \mathrm{~s}$, and the steadystate (rubbery modulus) information can be extrapolated with the proposed model if needed. The thickness of the cartilage samples is determined with needle probe techniques. A hy- 
Fig. 3 CETR UMT3 tribometer fitted with a 12-mm rigid indenter
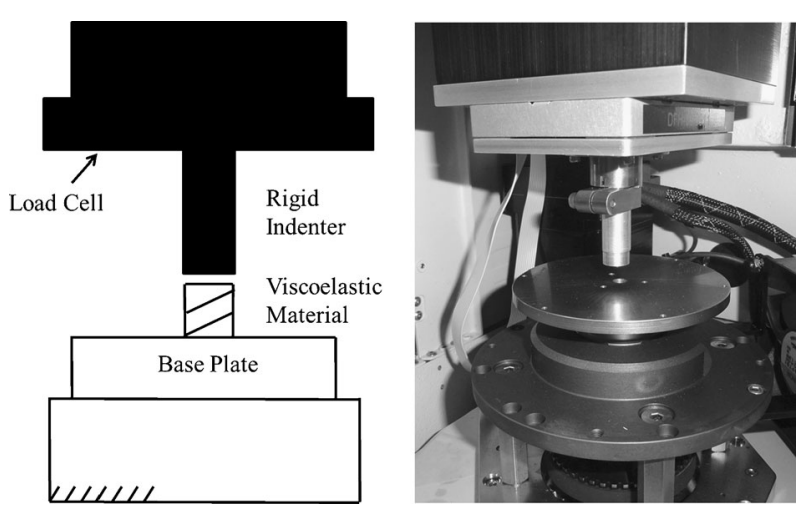

podermic needle is attached to the tribometer and travels through the cartilage plug. The locations of first contact, surface puncture, and contact with subchondral bone are clearly shown in the resulting force/displacement graphs. Each test yields a thickness measurement. Multiple measurements are averaged to give a mean thickness, which determines the strain in each sample. The strain is needed to calculate the modulus (discussed herein).

The stress-relaxation experiment is particularly useful because it contains a wide spectrum of storage and loss properties. Gurtin and Sternberg (1962) developed a constitutive law relating stress, strain and the relaxation modulus using Boltzmann's superposition principle. The viscoelastic model is time-dependent and retains memory of the material and loading history:

$$
\sigma(t)=\epsilon(0) E(t)+\int_{0}^{t} \dot{\epsilon}(\tau) E(t-\tau) d \tau,
$$

where $\sigma(t)$ is the stress, $\epsilon(t)$ is the strain, and $E(t)$ is the relaxation modulus. Typically, $\sigma(t)$ and $\epsilon(t)$ are either set or measured during experimentation, whereas $E(t)$ is obtained from a fixed strain input $\epsilon=\epsilon_{\text {step }}$ such that $E(t)=\sigma(t) / \epsilon_{\text {step }}$. The parameters of stress, strain, and elastic modulus in Eq. (1) are time-dependent. It should be noted that Eq. (1) describes a linear relationship between the strain history and the current stress. Transforming Eq. (1) into the Laplace domain allows for simple treatment of the convolution integral:

$$
\sigma(s)=s E(s) \epsilon(s)
$$

Equation (2) is similar to Hooke's law in the Laplace domain (for the uniaxial case). This provides the foundation for the elastic-viscoelastic correspondence principle.

To transfer between the Laplace and frequency domains, the Laplace parameter $s$ in Eq. (2) is replaced with $i \omega$, where $i$ is defined as $\sqrt{-1}$, and $\omega$ is the frequency in $\mathrm{rad} / \mathrm{s}$. Hence, applying $s \rightarrow i \omega$, Eq. (2) becomes

$$
\sigma(\omega)=(i \omega) E(\omega) \epsilon(\omega) \triangleq E^{*}(\omega) \epsilon(\omega)
$$

$E^{*}(\omega)$ is defined as the complex modulus $E^{*}(\omega)=(i \omega) E(\omega)$ and has two components, a real and an imaginary:

$$
E^{*}(\omega)=E^{\prime}(\omega)+i E^{\prime \prime}(\omega)
$$


Fig. 4 Physical representation of Prony series and fractional model

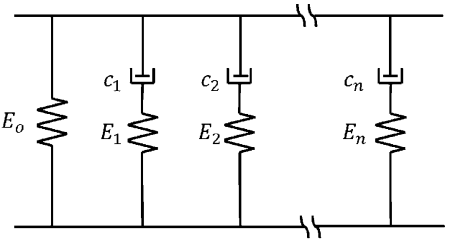

(a) Prony series

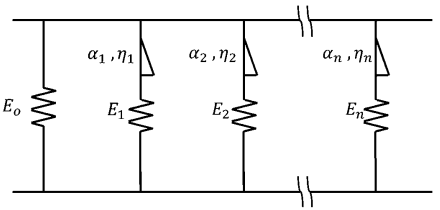

(b) Fractional calculus model

The real component $\left(E^{\prime}\right)$ is known as the storage modulus, whereas the imaginary component $\left(E^{\prime \prime}\right)$ is the loss modulus. Both measures describe the dynamic behavior (frequency dependency) of the material, in this case the cartilage. The correspondence principle is powerful because one constitutive formulation determines the amount of energy retained (stored) or lost (loss). Stress-strain constitutive equations must be formed that accurately model the experimental data. One such powerful formulation is the fractional calculus model.

To draw a comparison between the fractional calculus model and more common viscoelastic models, spring and dashpot systems are considered. Spring and dashpot systems contain both elastic and dissipative mechanisms simultaneously (Gurtin and Sternberg 1962; Szumski and Green 1991; Miller and Green 1997), which make them appropriate for modeling viscoelastic substances. There are many configurations of spring and damper systems, including the Maxwell, Kelvin-Voigt, and Prony series models. The Prony series (Fig. 4(a)) is particularly adept in stress-relaxation. However, it often requires many elements to adequately model the rapid relaxation seen in cartilage. The fractional calculus model has similarities with the Prony series, but the fractional model significantly reduces the number of elements and thus terms needed to characterize viscoelastic behavior. The model replaces the dashpot of each Maxwell element with a fractional "spring-pot," as seen in Fig. 4(b). The spring-pot interpolates between spring and dashpot behavior; in fact, the spring-pot is physically represented as ladder structure of springs and dashpots (Schiessel and Blumen 1993, 1995; Schiessel et al. 1995). Adjusting the constants of the ladder's "rungs" yields any fractional derivative $\alpha$ between 0 and 1. This gives the fractional model great flexibility. A spring-pot is mathematically described as

$$
\sigma_{\mathrm{sp}}=\eta \frac{d^{\alpha} \epsilon_{\mathrm{sp}}}{d t^{\alpha}}
$$

where $\alpha$ is a rational number between 0 and 1 , and $\eta$ is a material parameter similar to a damping coefficient. The parameter $\eta$ takes on nonstandard units that are similar to viscosity, $\mathrm{Pa} \mathrm{s}^{\alpha}$. The interpolative nature of the spring-pot element is clear: if $\alpha=0$, then the element becomes a spring, and if $\alpha=1$, then the element becomes a common viscous dashpot. For any $\alpha$ between 0 and 1, the element has both spring and dashpot behavior, as described by the hierarchical analogue (or ladder model) (Schiessel and Blumen 1993, 1995; Schiessel et al. 1995). The virtual increase in mathematical complexity of the fractional model is mitigated by significantly reducing the total terms that are needed to describe the stress/strain relationship. In comparison to the Prony series, fractional models typically require many 
fewer elements to obtain a similar quality of fit (e.g., in the current study, the fractional model uses $1 / 4$ of the elements of the Prony series). Applying fractional calculus to stressrelaxation, the relaxation modulus can be found in the frequency domain (detailed in the Appendix):

$$
E(\omega)=\frac{E_{0}}{i \omega}+\sum_{n=1}^{\infty} \frac{E_{n}(i \omega)^{\alpha}}{\left[(i \omega)^{\alpha}+\frac{E_{n}}{\eta_{n}}\right]}\left(\frac{1}{i \omega}\right) .
$$

In the time-domain, the fractional relaxation modulus is (Bagley 1989; Kisela 2009)

$$
E(t)=E_{0}+\sum_{n=1}^{\infty} E_{n} E_{\alpha}\left(-\frac{E_{n}}{\eta_{n}} t^{\alpha_{n}}\right),
$$

where $E_{\alpha}$ is the Mittag-Leffler function (Erdelyi et al. 1955),

$$
E_{\alpha, \beta}(z)=\sum_{k=0}^{\infty} \frac{z^{k}}{\Gamma(\alpha k+\beta)},
$$

and $\Gamma$ is the gamma function, $\Gamma(x)=(x-1)$ !.

Whereas the above expression provides a formal time-domain representation, practically the fractional model is difficult to fit in the time-domain because of convergence issues. To be practical in modeling, a finite number of terms must be used in the summation in Eq. (8). If the parameter $(\eta)$ is very small, then $k$ must be very large, and convergence issues arise. Typically, the fractional model is fit in the frequency domain to circumvent the Mittag-Leffler function; however, the work of Podlubny (1998) proposes a Matlab procedure for the Mittag-Leffler function to be fit in the time-domain. The Mittag-Leffler function is avoided altogether in the special case where $\alpha=1 / 2$. In this case, the Mittag-Leffler function reduces, and the fractional derivative model effectively becomes a complementary error function model (CERF) (Szumski and Green 1991; Szumski 1993) (detailed also in the Appendix). The complementary error function model is a robust and convenient model for viscoelasticity. It has the advantage of offering a concise time-domain solution in the form of the complementary error function multiplied by an exponential function given by

$$
E(t)=E_{0}+\sum_{n=1}^{\infty} E_{n} e^{\left(\mu_{n} \sqrt{t}\right)^{2}} \operatorname{erfc}\left(\mu_{n} \sqrt{t}\right),
$$

where $E_{n}$ and $\mu_{n}$ are material properties, and

$$
\mu_{n}=\frac{E_{n}}{\eta_{n}} .
$$

The complementary error function decays at a faster rate than the exponential increases, giving an overall relaxation behavior. The importance of the CERF model is its time-domain representation. Models with time-domain representations have utility in fitting noisy experimental data. The CERF model combines the advantages of the spring and damper models (simple mathematics) with the advantages of the fractional model. This is a very powerful formulation, which robustly models cartilage with as few as three constants: $E_{0}, E_{1}$, and $\mu_{1}$ (i.e., only one term $(n=1)$ in the summation Eq. (9)). Here, $\mu_{n}$ has nonstandard, but 
straightforward, units of $\mathrm{s}^{-1 / 2}$. Certain definitions such as a fractional time constant can be used to define a dimensionless relaxation curve, but the CERF model is simple enough to justify the units of $\mu_{n}$. The complementary error function can be computed by most engineering software packages. However, the main advantage of Eq. (9) is that it can be further reduced with an expansion given by Abramowitz and Stegun (1972):

$$
\begin{gathered}
\operatorname{erfc}(x)=\left(a_{1} y+a_{2} y^{2}+a_{3} y^{3}+a_{4} y^{4}+a_{5} y^{5}\right) e^{-x^{2}}, \\
y=\frac{1}{1+p x},
\end{gathered}
$$

where

$$
\begin{aligned}
& p=0.3275911, \quad a_{1}=0.254829592, \quad a_{2}=-0.284496736, \\
& a_{3}=1.421413741, \quad a_{4}=-1.453152027, \quad a_{5}=1.061405429 .
\end{aligned}
$$

The maximum error of this expansion is $1.5 \times 10^{-7}$. The advantage of using Eq. (11) to represent the $\operatorname{erfc}(\cdot)$ in Eq. (9) is that the exponentials cancel out as $x=\mu \sqrt{t}$. The result is a simple polynomial expression:

$$
\begin{gathered}
E(t)=E_{0}+\sum_{n=1}^{\infty} E_{n}\left(a_{1} y+a_{2} y^{2}+a_{3} y^{3}+a_{4} y^{4}+a_{5} y^{5}\right), \\
y=\frac{1}{1+\mu p \sqrt{t}} .
\end{gathered}
$$

Fitting the time-domain relaxation modulus using Eq. (13) is straightforward. Only a simple least-squares algorithm is needed to determine the parameters of $E_{n}$ and $\mu_{n}$ for $n=1,2,3, \ldots, n$. The CERF model is suitable for any general viscoelastic behavior and is not specifically a biphasic model; however, it is capable of capturing the relaxation behavior of biphasic materials very well.

The time-domain is important for performing experiments and qualitative understanding of stress-relaxation, but the frequency domain is fundamental to a dynamic analysis. However, transferring the quite noisy raw experimental signal from the time to the frequency domain (using a fast Fourier transform or other techniques) is challenging, even with filtering and smoothing algorithms (Smyth 2013). The CERF model does not require such a manipulation since there are convenient formulations in both the time and frequency domains. Once the parameters $E_{n}$ and $\mu_{n}$ are obtained from the time-domain fit, the analytic form of the CERF is readily available in the frequency domain (see the Appendix):

$$
E(\omega)=\frac{E_{0}}{i \omega}+\sum_{n=1}^{\infty} \frac{E_{n}(i \omega)^{1 / 2}}{\left[(i \omega)^{1 / 2}+\frac{E_{n}}{\eta_{n}}\right]}\left(\frac{1}{i \omega}\right) .
$$

The storage and loss moduli are given as a function of frequency $\omega$ :

$$
E^{\prime}=E_{0}+\sum_{n=1}^{\infty} E_{n}\left[\frac{\left(\frac{\sqrt{2 \omega}}{2}\right) \mu_{n}+\omega}{\mu_{n}^{2}+\mu_{n} \sqrt{2 \omega}+\omega}\right],
$$


Table 1 Comparison data for CERF and Prony series $(n=1)$

\begin{tabular}{lll}
\hline & CERF & Prony \\
\hline$E_{0}(\mathrm{MPa})$ & 1.409 & 0.774 \\
$E_{1}(\mathrm{MPa})$ & 0.549 & 0.677 \\
$\mu_{1}^{2}$ or $\lambda_{1}(1 / \mathrm{s})$ & $\mu_{1}^{2}=0.8649 \times 10^{-2}$ & $\lambda_{1}=0.410 \times 10^{-1}$ \\
\hline & $E^{\prime \prime}=\sum_{n=1}^{\infty} E_{n}\left[\frac{\left(\frac{\sqrt{2 \omega}}{2}\right) \mu_{n}}{\mu_{n}^{2}+\mu_{n} \sqrt{2 \omega}+\omega}\right]$.
\end{tabular}

\section{Results}

The CERF model ( $n=1$, Eq. (13)) is fit to the relaxation behavior, which is pronounced in the initial time of the experiment (Fig. 5(a)). In addition, the Prony series model (Eq. (18), $n=1$ ) is also shown in Fig. 5(a) as a comparison to the CERF model (see Table 1 for fit parameters).

$$
E_{\text {Prony }}(t)=E_{0}+\sum_{n=1}^{\infty} E_{n} e^{-\lambda_{n} t} .
$$

The Prony model is fit in a similar least-squares manner and contains the same number of constants as the CERF. When $n=1$, the Prony series is known as the standard linear solid (Smyth 2013). Clearly, the standard linear model is unable to capture the relaxation in the first $100 \mathrm{~s}$ of decay, rendering it of little use in the current study. The relaxation behavior is captured well with the CERF model ( $n=1$, which is the CERF equivalent to a standard linear solid). Only minor deviation between the fit and the actual data is seen in both scales in Fig. 5. The deviation between the model and experimental data corresponds to the highest frequency information. For horses (and humans), frequency ranges greater than $4-5 \mathrm{~Hz}$ are not accessed during even the most strenuous exercises. Therefore, it is less important to capture this corresponding region of the relaxation data, i.e., from $t=0 \mathrm{~s}$ to $t=200 \mathrm{~ms}$. The CERF robustly models the important decades of relaxation behavior (shown in Fig. 5(b) using a semi-log scale), which makes its utility apparent for biological materials. The CERF model is powerful in that only three parameters, $E_{0}, E_{1}$, and $\mu_{1}$ (corresponding to $n=1$ ), are needed to robustly fit the bulk relaxation behavior. In applications where biphasic materials are integrated into larger system dynamics, this compact model has great utility.

The frequency domain allows for study of cartilage as a function of gait. The elasticviscoelastic correspondence principle transfers time-dependent information to the Laplace and frequency domains without loss of generality. Therefore, stress-relaxation experiments give directly the storage and loss moduli as functions of frequency $\omega$. A comparison of the CERF and Prony series models $(n=1)$ in the frequency domain is shown in Fig. 6 for the storage and loss modulus. The two models have significant differences over many frequency decades. Considering the storage modulus (Fig. 6(a)), the CERF and Prony models display large differences, particularly at low frequencies. Likewise, the loss modulus (Fig. 6(b)) has major differences (nearly an order of magnitude in the physiological range), indicating that the two models are fundamentally different. Based on the relaxation seen in Fig. 5, the CERF describes the frequency behavior of cartilage more accurately. 
Fig. 5 Example fit of CERF to stress-relaxation experiment of cartilage plug immersed in saline

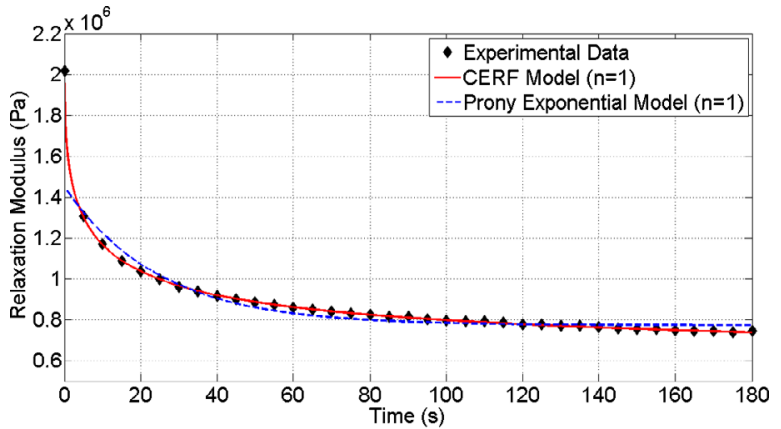

(a) CERF and Prony fit $(n=1)$ and to typical experimental data (linear-scale)

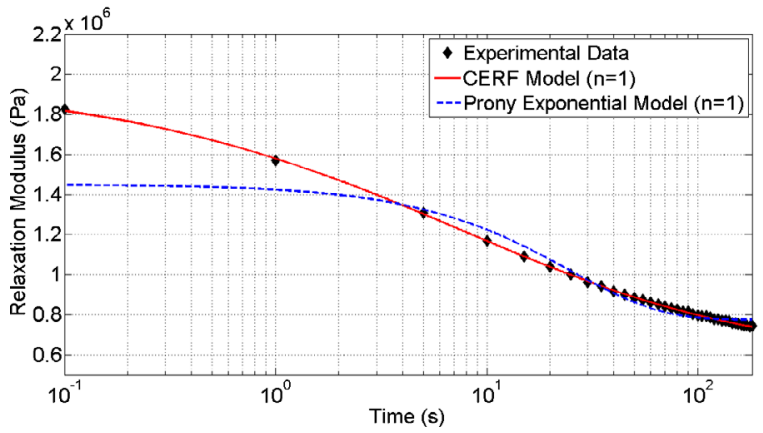

(b) CERF and Prony fit $(n=1)$, displayed on a semi-log scale

Analysis in the frequency domain shows that cartilage has strong frequency characteristics. Common gaits for cartilage are in the viscoelastic transition region, which is between the higher frequencies giving the glassy region $(4 \mathrm{~Hz})$ and the lower frequencies giving the rubbery region $(0.25 \mathrm{~Hz})$. Cartilage adjusts to a stimulus by storing and dissipating different amounts of energy, depending on the frequency of perturbation. This is potentially an important characteristic of biphasic materials. The adaptive nature of cartilage is well suited for bioinspired designs of bearings and dampers.

The thickness of the cartilage plugs is not known a priori. This complicates the analysis as the testing procedure imposed a predetermined displacement on the cartilage sample. The strain is determined by the thickness of the sample. Therefore, results obtained from the relaxation experiments are inherently over a range of strains. Attempts were made to limit the strains to 5-15\%; however, there are a few cases where $15 \%$ is exceeded. Each measurement is fit with Eq. (9) to determine the parameters $E_{0}, E_{1}$, and $\mu_{1}$. An example fit of the CERF $(n=1)$ to actual cartilage relaxation data is shown in Fig. 5. When $t=0$, the glassy modulus is obtained ( $E_{\text {glassy }}=E_{0}+E_{1}$ ), and as $t \rightarrow \infty$, the rubbery modulus is found $\left(E_{\text {rubbery }}=E_{0}\right)$. These quantities are reported in the vertical columns of Table 2 . Student's t test is used to provide upper and lower bounds for the respective moduli. It is difficult to generalize all of the samples as one conglomerate; however, this is provided as an estimate of the glassy and rubbery moduli. The combined results represent a range of likely cartilage behavior, and each sample is said to have certain strain-dependent properties. More exhaustive testing and additional data should be used to corroborate this finding. The large 
Fig. 6 Comparison of the CERF and Prony models in the frequency domain

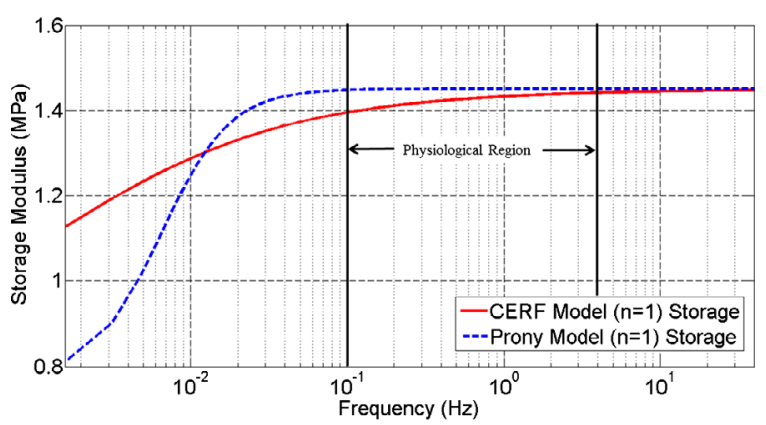

(a) The storage modulus of the $(n=1)$ CERF and Prony models

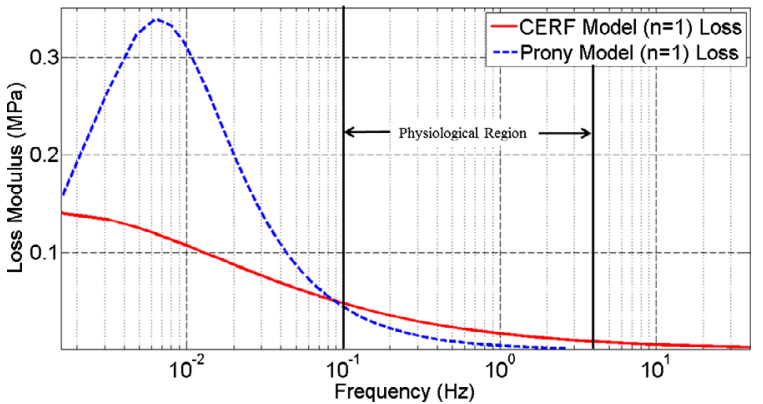

(b) The loss modulus of the $(\mathrm{n}=1) \mathrm{CERF}$ and Prony models

standard deviation and error seen in Table 2 is likely due to a relatively small sample size and large variability in samples due to age, breed, use, etc.

Large variations are expected in biological samples. Each cartilage explant is unique, which increases the difficulty of drawing meaningful conclusions from the data. Genetics, weight, age, diet, gender, and use can influence the mechanical properties of cartilage. The model parameters obtained from experiments are expected to have large variations. However, a prevailing trend is that the transition period of cartilage coincides with the physiological range of exercise. At lower frequencies, cartilage dissipates more energy than at higher frequencies, where additional elasticity is available in the joints. The transition range of cartilage occurs in the middle of the common frequencies of motion $(0.25-4 \mathrm{~Hz})$. It is possible that the adaptive nature of cartilage is biologically designed for this purpose.

\section{Discussion}

A simple model that describes the behavior of cartilage is proposed. Under the specified loading (stress-relaxation), the CERF model is able to account for biphasic behavior when considered as a conglomerate material. Many additional tests are required to quantitatively describe the behavior of equine cartilage. The CERF model does have limitations; however, its application is appropriate in many situations. In particular, the CERF model has utility in impact studies, adaptive bearings and dampers design, and study of systems that include biphasic materials.

The preliminary results obtained should justify additional research and experimentation. More cartilage samples are needed for statistical significance; however, the experimental 
Table 2 Tabulated data for cartilage samples immersed in saline

\begin{tabular}{lll}
\hline Fluid bath & Saline & \\
Number of samples & 12 & \\
Degrees of freedom & 11 & \\
Strain range & $7.35-13.08 \%$ & Rubbery modulus (MPa) \\
\hline & Glassy modulus (MPa) & 0.323 \\
\hline Avg. & 1.240 & 0.226 \\
Std. & 1.011 & 0.065 \\
SE & 0.292 & \\
\hline Two-tailed, 95 \% Student's $t$ test & 2.201 & 0.143 \\
\hline Confidence interval \pm & 0.642 & 0.180 \\
Lower mean & 0.597 & 0.467 \\
Upper mean & 1.882 & \\
\hline
\end{tabular}

data is a proof-of-concept. The bulk mechanical response of articular cartilage in stressrelaxation can be described with the spring/spring-pot systems proposed. This is important for future analysis of biphasic materials, including biomimetic substances. The simplicity of the CERF model is beneficial in large-scale finite-element (or other) studies. Additional fractional models also exist that may have utility for cartilage. In particular, the recent anisotropic model from Hilton (2012) could be useful in cartilage mechanics.

The current work is focused on describing the stress-relaxation behavior of cartilage in unconfined compression. Many previous researchers have used stress-relaxation to determine properties for modeling, but a strictly phenomenological model has not been thoroughly explored. The majority of tests being performed are creep tests, which are typically easier to execute. However, stress-relaxation experiments are more analogous to movements experienced during exercise. The methods used herein are advantageous compared to previous studies because they more closely mimic biological function and require fewer material properties.

Equine articular cartilage displays similarities to human cartilage (Malda et al. 2012). Within the equine skeleton, the stifle joint is harvested for its regions of thick, flat cartilage and for the mechanical similarity to the human knee. A CETR-UMT-3 tribometer performs stress-relaxation experiments, which mimic biological function during exercise such as walking or running. The tribometer produces a nearly instantaneous displacement. The resulting relaxation in the cartilage is captured and fit phenomenologically. The pseudo spring and damper model (CERF) is studied in the time and frequency domains. The relationship between time and frequency domains is analytic. This is an important advantage of the phenomenological approach, and the Boltzmann convolution integral that governs the stress-strain relationships.

In the limited range of gaits associated with physiological movement, the storage and loss moduli of cartilage transition dramatically. This behavior is common to viscoelastic materials. Cartilage appears to adapt to the type of exercise undertaken. Although the many variables of genetics make it difficult to draw comparisons between samples, the transition region is ubiquitous. This could contribute to cartilage's longevity and may be an important design aspect for biomimetic materials. Ultimately, the CERF model provides a compact tool that can be used to evaluate biphasic material behavior if the phases do not require 
separation. The CERF is not a detailed model for cartilage, but it allows for practical analysis to be undertaken.

Unconfined compression tests and the CERF model can characterize cartilage with as few as two types of terms: $E$ and $\mu$. Poisson's ratio and other experimental "fudge-factors" are not required for fitting the data. For a complex material, models that can capture the majority of the mechanical response with relatively few parameters are very useful. The advantage of the phenomenological characterization is its simplicity. The techniques given can be used for comparisons between species or between healthy and diseased cartilage. The material properties given are needed for a full dynamic or impact study. Potential other uses of the CERF include: magnetic resonance elastography (MRE), biphasic bearing/damper evaluation, and control problems considering fractional order viscoelasticity.

\section{Conclusions}

There has been little previous work linking fractional calculus and biomechanics, and in particular, cartilage mechanics. However, as displayed in the current study, the robustness and flexibility of fractional calculus is well suited for such applications. The special case of fractional derivative, $\alpha=1 / 2$, has unique advantages for modeling stress-relaxation in cartilage. The time-domain representation of the CERF model (Eq. (13)) is very powerful and intrinsically useful when the elastic-viscoelastic correspondence principle is invoked. For the first time, it is shown that the relaxation modulus can be robustly expressed as a polynomial, and the polynomial expansion is easily fit in a least-squares sense. This alone is advantageous when compared to many models that present significant fitting challenges. The succinctness of the CERF model is a major factor in its utility. In applications where cartilage (or a biphasic material in general) is modeled as a component of a larger system, the CERF model is accurate without being inordinate. These reasons warrant additional study in fractional calculus and biomechanics.

Acknowledgements This work is supported by NSF Grant No. DGE-1148903.

\section{Appendix}

For simplicity, only a one-element fractional derivative model is developed. However, the one-element model can be generalized to include multiple elements in parallel by the principle of linear superposition. This concept is analogous to that of the more common Prony series. The constitutive equation relating stress to strain is similar to that of a standard linear viscoelastic material:

$$
\left(1+\frac{E_{0}}{E_{1}}\right) \frac{d \epsilon}{d t}+\frac{E_{0}}{c_{1}} \epsilon=\frac{1}{E_{1}} \frac{d \sigma}{d t}+\frac{1}{c_{1}} \sigma,
$$

except that the dashpot is replaced with the spring-pot element

$$
\frac{d \epsilon}{d t} \longleftarrow \frac{d^{\alpha} \epsilon}{d t^{\alpha}}
$$

and

$$
\frac{d \sigma}{d t} \longleftarrow \frac{d^{\alpha} \sigma}{d t^{\alpha}}
$$


leading to the constitutive equation for the fractional derivative model

$$
\left(1+\frac{E_{0}}{E_{1}}\right) \frac{d^{\alpha} \epsilon}{d t^{\alpha}}+\frac{E_{0}}{\eta_{1}} \epsilon=\frac{1}{E_{1}} \frac{d^{\alpha} \sigma}{d t^{\alpha}}+\frac{1}{\eta_{1}} \sigma .
$$

In Eq. (A.4), the damping coefficient $c_{1}$ has been replaced by $\eta_{1}$ to reflect unit consistency. If $\alpha=1$, then the constitutive model becomes the standard linear material (one-term Prony model shown in Eq. (A.1)), and the units of $\eta_{1}$ collapse to those of $c_{1}$ (Pas). If $\alpha=0$, then the spring-pot simply becomes strictly a spring, and the entire model is reduced to an equivalent linear spring. For any fractional valued $\alpha$ between 0 and 1 , the spring-pot element has both spring and dashpot behavior.

Equation (A.4) is conveniently analyzed in the Laplace domain, which allows for the treatment of the fractional power (taking Caputo's definition of the fractional-order derivative and assuming that the initial conditions for stress and strain can be set to zero (Podlubny 1998)):

$$
\left[\left(1+\frac{E_{0}}{E_{1}}\right) s^{\alpha}+\frac{E_{0}}{\eta_{1}}\right] \epsilon(s)=\left(\frac{1}{E_{1}} s^{\alpha}+\frac{1}{\eta_{1}}\right) \sigma(s) .
$$

Utilizing the elastic-viscoelastic correspondence principle (Eq. (2)), the relaxation modulus $E(s)$ can be found from Eq. (A.5):

$$
E(s)=\frac{\left[\left(1+\frac{E_{0}}{E_{1}}\right) s^{\alpha}+\frac{E_{0}}{\eta_{1}}\right]}{\left(\frac{1}{E_{1}} s^{\alpha}+\frac{1}{\eta_{1}}\right)} \frac{1}{s} .
$$

The relationship between the Laplace and frequency domains allows for the fractional model to be obtained:

$$
E(\omega)=\frac{\left[\left(1+\frac{E_{0}}{E_{1}}\right)(i \omega)^{\alpha}+\frac{E_{0}}{\eta_{1}}\right]}{\left[\frac{1}{E_{1}}(i \omega)^{\alpha}+\frac{1}{\eta_{1}}\right]}\left(\frac{1}{i \omega}\right) .
$$

With some algebra, Eq. (A.7) can be reduced to

$$
E(\omega)=\frac{1}{i \omega}\left[E_{0}+\frac{E_{1}(i \omega)^{\alpha}}{\left[(i \omega)^{\alpha}+\frac{E_{1}}{\eta_{1}}\right]}\right] .
$$

If required, the fractional model can be generalized to include more spring-pot elements:

$$
E(\omega)=\frac{1}{i \omega}\left[E_{0}+\sum_{n=1}^{\infty} \frac{E_{n}(i \omega)^{\alpha}}{\left[(i \omega)^{\alpha}+\frac{E_{n}}{\eta_{n}}\right]}\right] .
$$

Theoretically, an infinite number of terms can be used. In practice, this number is finite. The complex modulus is found from the elastic-viscoelastic correspondence principle (Eq. (2)):

$$
E^{*}(\omega)=E_{0}+\sum_{n=1}^{\infty} \frac{E_{n}(i \omega)^{\alpha}}{\left[(i \omega)^{\alpha}+\frac{E_{n}}{\eta_{n}}\right]} .
$$

Simplifications for $\alpha=1 / 2$ (special case)

For the special case of $\alpha=1 / 2$, the mathematics of the fractional model simplify dramatically. In the time domain, a concise solution appears in the form of a complementary 
error function multiplied by a decaying exponential. An analytic form of the model can be found for the frequency domain solution as well. Consider a one-term fractional model with $\alpha=1 / 2$ :

$$
E^{*}(\omega)=E_{0}+\frac{E_{1}(i \omega)^{1 / 2}}{\left[(i \omega)^{1 / 2}+\frac{E_{1}}{\eta_{1}}\right]} .
$$

The square root of $i \omega$ can be found from the generalized form of de Moivre's theorem:

$$
(i \omega)^{1 / 2}=\frac{\sqrt{2 \omega}}{2}(1+i) .
$$

Two substitutions help clarify the mathematics:

$$
\begin{aligned}
& \beta=\frac{\sqrt{2 \omega}}{2}, \\
& \mu_{1}=\frac{E_{1}}{\eta_{1}} .
\end{aligned}
$$

Both $\beta$ and $\mu_{1}$ have the units $\mathrm{s}^{-1 / 2}$. Substituting these relations into Eq. (A.11) yields

$$
E^{*}(\omega)=E_{0}+\frac{E_{1} \beta(1+i)}{\mu_{1}+\beta(1+i)} .
$$

After algebraic manipulation and the simplification $(1+i)^{2}=2 i$, Eq. (A.15) is

$$
E^{*}(\omega)=E_{0}+E_{1} \beta\left[\frac{\mu_{1}-\left(2 \beta-\mu_{1}\right) i}{\mu_{1}^{2}-2 \beta^{2} i}\right] .
$$

Additional manipulation leads to a usable expression:

$$
E^{*}(\omega)=E_{0}+E_{1} \beta\left[\frac{\mu_{1}+2 \beta}{\mu_{1}^{2}+2 \mu_{1} \beta+2 \beta^{2}}+\frac{i \mu_{1}}{\mu_{1}^{2}+2 \mu_{1} \beta+2 \beta^{2}}\right] .
$$

Reintroducing the substitution of Eq. (A.13), the derivation of the one-element CERF model is complete:

$$
E^{*}(\omega)=E_{0}+E_{1}\left[\frac{\left(\frac{\sqrt{2 \omega}}{2}\right) \mu_{1}+\omega}{\mu_{1}^{2}+\mu_{1} \sqrt{2 \omega}+\omega}+\frac{i\left(\frac{\sqrt{2 \omega}}{2}\right) \mu_{1}}{\mu_{1}^{2}+\mu_{1} \sqrt{2 \omega}+\omega}\right] .
$$

Equations (A.18) can be generalized for any number of fractional terms, although the utility of the fractional model is that few terms typically need to be used to characterize viscoelastic behavior:

$$
E^{*}(\omega)=E_{0}+\sum_{n=1}^{\infty} E_{n}\left[\frac{\left(\frac{\sqrt{2 \omega}}{2}\right) \mu_{n}+\omega}{\mu_{n}^{2}+\mu_{n} \sqrt{2 \omega}+\omega}+\frac{i\left(\frac{\sqrt{2 \omega}}{2}\right) \mu_{n}}{\mu_{n}^{2}+\mu_{n} \sqrt{2 \omega}+\omega}\right]
$$

and if

$$
E^{*}(\omega)=E^{\prime}(\omega)+i E^{\prime \prime}(\omega),
$$


then

$$
\begin{gathered}
E^{\prime}(\omega)=E_{0}+\sum_{n=1}^{\infty} E_{n}\left[\frac{\left(\frac{\sqrt{2 \omega}}{2}\right) \mu_{n}+\omega}{\mu_{n}^{2}+\mu_{n} \sqrt{2 \omega}+\omega}\right], \\
E^{\prime \prime}(\omega)=\sum_{n=1}^{\infty} E_{n}\left[\frac{\left(\frac{\sqrt{2 \omega}}{2}\right) \mu_{n}}{\mu_{n}^{2}+\mu_{n} \sqrt{2 \omega}+\omega}\right] .
\end{gathered}
$$

For the fractional derivative model where $\alpha=1 / 2$, there exists a concise time-domain solution (Szumski and Green 1991):

$$
E(t)=E_{0}+\sum_{n=1}^{\infty} E_{n} e^{\left(\mu_{n}^{2} t\right)} \operatorname{erfc}\left(\mu_{n} \sqrt{t}\right),
$$

which is a decaying complementary error function multiplied by an increasing exponential. The time-domain solution is critical for fitting experimental data. The Laplace transformation of Eq. (9) is given by Szumski and Green (1991):

$$
E(s)=\frac{1}{s}\left[E_{0}+\sum_{n=1}^{\infty} \frac{E_{n} \sqrt{s}}{\sqrt{s}+\mu_{n}}\right] .
$$

Equation (A.24) and application of the elastic-viscoelastic correspondence principle allows us to relate the CERF model in the time and frequency domains, noting the connection between the Laplace and Fourier transformations (replace the Laplace variable $s$ with the Fourier variable $i \omega$ ). We then arrive back at Eq. (A.19).

\section{References}

Erdelyi, A., Oberhettinger, F., Magnus, W., Tricomi, F. (eds.): Higher Transcendental Functions, vol. III. McGraw-Hill, New York (1955)

Abramowitz, M., Stegun, I.A. (eds.): Handbook of Mathematical Functions. Dover, New York (1972)

Argatov, I.I.: Mathematical modeling of linear viscoelastic impact: application to drop impact testing of articular cartilage. Tribol. Int. 63, 213-225 (2013)

Armstrong, C.G., Lai, W.M., Mow, V.C.: An analysis of the unconfined compression of articular cartilage. J. Biomech. Eng. 106(2), 165-173 (1984)

Ateshian, G.A.: The role of interstitial fluid pressurization in articular cartilage lubrication. J. Biomech. 42(9), 1163-1176 (2009)

Ateshian, G.A., Warden, W.H., Kim, J.J., Grelsamer, R.P., Mow, V.C.: Finite deformation biphasic material properties of bovine articular cartilage from confined compression experiments. J. Biomech. 30(11-12), 1157-1164 (1997)

Ateshian, G.A., Wang, H., Lai, W.M.: The role of interstitial fluid pressurization and surface porosities on the boundary friction of articular cartilage. J. Tribol. 120(2), 241-248 (1998)

Bagley, R.L.: Power law and fractional calculus model of viscoelasticity. AIAA J. 27(10), 1412-1417 (1989)

Bagley, R.L., Torvik, P.J.: A generalized derivative model for an elastomer damper. Shock Vibr. Bull. 49(2), 135-143 (1979)

Bagley, R.L., Torvik, P.J.: A theoretical basis for the application of fractional calculus to viscoelasticity. J. Rheol. 27(3), 201-210 (1983).

Bagley, R.L., Torvik, P.J.: Fractional calculus in the transient analysis of viscoelastically damped structures. AIAA J. 23(6), 918-925 (1985)

Bagley, R.L., Torvik, P.J.: On the fractional calculus model of viscoelastic behavior. J. Rheol. 30(1), $133-155$ (1986) 
Carpinteri, A., Mainardi, F.: Fractals and Fractional Calculus in Continuum Mechanics. Courses and Lectures/International Centre for Mechanical Sciences/International Centre for Mechanical Sciences Udine, vol. 378. Springer, London (1997)

Charnley, J.: The lubrication of animal joints in relation to surgical reconstruction by arthroplasty. Ann. Rheum. Dis. 19, 10-19 (1960)

Coletti, J.M., Akeson, W.H., Woo, S.L.Y.: A comparison of the physical behavior of normal articular cartilage and the arthroplasty surface. J. Bone Jt. Surg. 54-A(1), 147-160 (1972)

DiSilvestro, M.R., Suh, J.K.F.: A cross-validation of the biphasic poroviscoelastic model of articular cartilage in unconfined compression, indentation, and confined compression. J. Biomech. 34(4), 519-525 (2001)

Ehlers, W., Markert, B.: A linear viscoelastic two-phase model for soft tissues: application to articular cartilage. Z. Angew. Math. Mech. 80(S1), 149-152 (2000)

Ehlers, W., Markert, B.: A linear viscoelastic biphasic model for soft tissues based on the theory of porous media. J. Biomech. Eng. 123(5), 418-424 (2001)

Eisenfeld, J., Mow, V.C., Lipshitz, H.: Mathematical analysis of stress relaxation in articular cartilage during compression. Math. Biosci. 39(1-2), 97-112 (1978)

Elsharkawy, A.A., Nassar, M.M.: Hydrodynamic lubrication of squeeze-film porous bearings. Acta Mech. 118, 121-134 (1996)

Friswell, M.: The response of rotating machines on viscoelastic supports. Int. Rev. Mec. Eng. 1(1), 32-40 (2007)

Fung, Y.C.: Elasticity of soft tissues in simple elongation. Am. J. Physiol. 213(6), 1532-1544 (1967)

Garcia, J.J., Cortes, D.H.: A nonlinear biphasic viscohyperelastic model for articular cartilage. J. Biomech. 39(16), 2991-2998 (2006)

Grybos, G.R.: The dynamics of a viscoelastic rotor in flexible bearings. Arch. Appl. Mech. 61(1), 479-487 (1991)

Gurtin, M.E., Sternberg, E.: On the linear theory of viscoelasticity. Arch. Ration. Mech. Anal. 11(1), 291-356 (1962)

Hilton, H.H.: Generalized fractional derivative anisotropic viscoelastic characterization. Materials 5(1), 169191 (2012). doi:10.3390/ma5010169

Julkunen, P., Wilson, W., Jurvelin, J.S., Rieppo, J., Qu, C.J., Lammi, M.J., Korhonen, R.K.: Stress relaxation of human patellar articular cartilage in unconfined compression: prediction of mechanical response by tissue composition and structure. J. Biomech. 41(9), 1978-1986 (2008)

Kisela, T.: Fractional generalization of the classical viscoelasticity models. In: Proceedings of 8th International Conference Aplimat 2009, pp. 593-600 (2009)

Koeller, R.: Applications of fractional calculus to the theory of viscoelasticity. J. Appl. Mech. 51, 299-307 (1984)

Koeller, R.C.: Polynomial operators, Stieltjes convolution, and fractional calculus in hereditary mechanics. Acta Mech. 58(3-4), 251-264 (1986)

Lai, W.M., Mow, V.C., Roth, V.: Effects of nonlinear strain-dependent permeability and rate of compression on the stress behavior of articular cartilage. J. Biomech. Eng. 103(2), 61-66 (1981)

Lai, W.M., Hou, J.S., Mow, V.C.: A triphasic theory for the swelling and deformation behaviors of articular cartilage. J. Biomech. Eng. 113(3), 245-258 (1991)

Lakes, R.: Viscoelastic Solids. Mechanical and Aerospace Engineering Series. Taylor \& Francis, London (1998)

Magin, R.: Fractional Calculus in Bioengineering. Begell House Publishers, Readding (2006)

Mainardi, F., Spada, G.: Creep, relaxation and viscosity properties for basic fractional models in rheology. Eur. Phys. J. Spec. Top. 193(1), 133-160 (2011)

Mak, A.F.: The apparent viscoelastic behavior of articular cartilage-the contributions from the intrinsic matrix viscoelasticity and interstitial fluid flows. J. Biomech. Eng. 108(2), 123-130 (1986)

Malda, J., Benders, K.E.M., Klein, T.J., de Grauw, J.C., Kik, M.J.L., Hutmacher, D.W., Saris, D.B.F., van Weeren, P.R., Dhert, W.J.A.: Comparative study of depth-dependent characteristics of equine and human osteochondral tissue from the medial and lateral femoral condyles. Osteoarthr. Cartil. 20(10), 11471151 (2012)

McCutchen, C.W.: The frictional properties of animal joints. Wear 5(1), 1-17 (1962)

Miller, B., Green, I.: On the stability of gas lubricated triboelements using the step jump method. J. Tribol. 119(1), 193-199 (1997)

Mow, V., Gu, W., Chen, F.: Structure and Function of Articular Cartilage and Meniscus. In: Basic Orthopaedic Biomechanics \& Mechano-Biology, 3rd edn., pp. 181-258. Lippincott Williams \& Wilkins, Philadelphia (2005)

Mow, V.C., Mansour, J.M.: The nonlinear interaction between cartilage deformation and interstitial fluid flow. J. Biomech. 10(1), 31-39 (1977) 
Mow, V.C., Lipshitz, H., Glimcher, M.J.: Mechanisms for stress relaxation in articular cartilage. In: 23rd Annual Meeting of the Orthopaedic Research Society, Las Vegas, vol. 2, p. 71. The Orthopaedic Research Society, Rosemont (1977)

Mow, V.C., Kuei, S.C., Lai, W.M., Armstrong, C.G.: Biphasic creep and stress relaxation of articular cartilage in compression: theory and experiments. J. Biomech. Eng. 102(1), 73-84 (1980)

Mow, V.C., Ateshian, G.A., Spilker, R.L.: Biomechanics of diarthrodial joints: a review of twenty years of progress. J. Biomech. Eng. 115(4B), 460-467 (1993)

Parsons, J.R., Black, J.: The viscoelastic shear behavior of normal rabbit articular cartilage. J. Biomech. 10(1), 21-29 (1977)

Podlubny, I.: Fractional Differential Equations: An Introduction to Fractional Derivatives, Fractional Differential Equations, to Methods of Their Solution and Some of Their Applications. Mathematics in Science and Engineering, Elsevier, Amsterdam (1998)

Rogers, L.: Operators and fractional derivatives for viscoelastic constitutive equations. J. Rheol. 27(4), 351372 (1983)

Schiessel, H., Blumen, A.: Hierarchical analogues to fractional relaxation equations. J. Phys. A, Math. Gen. 26(19), 5057 (1993)

Schiessel, H., Blumen, A.: Mesoscopic pictures of the sol-gel transition: ladder models and fractal networks. Macromolecules 28(11), 4013-4019 (1995). http://pubs.acs.org/doi/pdf/10.1021/ma00115a038. doi: $10.1021 / \mathrm{ma} 00115 \mathrm{a} 038$

Schiessel, H., Metzler, R., Blumen, A., Nonnenmacher, T.F.: Generalized viscoelastic models: their fractional equations with solutions. J. Phys. A, Math. Gen. 28(23), 6567 (1995)

Simon, B.R., Coats, R.S., Woo, S.L.Y.: Relaxation and creep quasilinear viscoelastic models for normal articular cartilage. J. Biomech. Eng. 106(2), 159-164 (1984)

Smyth, P.: Viscoelastic behavior of articular cartilage in unconfined compression. Master's thesis, Georgia Institute of Technology (2013)

Smyth, P.A., Rifkin, R.E., Jackson, R.L., Reid Hanson, R.: The average roughness and fractal dimension of articular cartilage during drying. Scanning 36(3), 368-375 (2014)

Szumski, R.G.: A finite element formulation for the time domain vibration analysis of an elastic-viscoelastic structure. Ph.D. thesis, Georgia Institute of Technology (1993)

Szumski, R.G., Green, I.: Constitutive laws in time and frequency domains for linear viscoelastic materials. J. Acoust. Soc. Am. 90(40), 2292 (1991)

Tanaka, E., Pelayo, F., Kim, N., Lamela, M.J., Kawai, N., Fernãndez-Canteli, A.: Stress relaxation behaviors of articular cartilages in porcine temporomandibular joint. J. Biomech. 47(7), 1582-1587 (2014)

Torvik, P.J., Bagley, R.L.: On the appearance of the fractional derivative in the behavior of real materials. J. Appl. Mech. 51(2), 294-298 (1984)

Wang, J.L., Parnianpour, M., ShiraziAdl, A., Engin, A.E.: Failure criterion of collagen fiber: viscoelastic behavior simulated by using load control data. Theor. Appl. Fract. Mech. 27(1), 1-12 (1997)

West, B., Bologna, M., Grigolini, P.: Physics of Fractal Operators. Institute for Nonlinear Science/Springer, Berlin (2003)

Wilson, W., van Donkelaar, C.C., van Rietbergen, B., Ito, K., Huiskes, R.: Stresses in the local collagen network of articular cartilage: a poroviscoelastic fibril-reinforced finite element study. J. Biomech. 37(3), 357-366 (2004)

Wilson, W., van Donkelaar, C.C., van Rietbergen, B., Huiskes, R.: A fibril-reinforced poroviscoelastic swelling model for articular cartilage. J. Biomech. 38(6), 1195-1204 (2005)

Woo, S.L.Y., Simon, B.R., Kuei, S.C., Akeson, W.H.: Quasi-linear viscoelastic properties of normal articular cartilage. J. Biomech. Eng. 102(2), 85-90 (1980) 